\title{
Magnetic Data Analysis for the ALS Lattice Magnets
}

\author{
Roderich Keller \\ Lawrence Berkeley Laboratory 80-101 \\ Berkeley, CA 94720
}

\begin{abstract}
The Advanced Light Source (ALS), now under construction at Lawrence Berkeley Laboratoiy, is a third-generation synchrotron radiation source designed to produce extremely bright photon beams in the UV and soft X-ray regions [1]. The lattice magnets to be installed in the two rings, a $1-1.9 \mathrm{GeV}$ storage ring, and a $1.5 \mathrm{GeV}$ booster synchrotron, have been magnetically measured to ascertain their excitation curves and field uniformity. In this paper the analysis of these data is discussed, and results for measured magnets are given.
\end{abstract}

\section{INTRODUCTION}

The magnets discussed in this paper are parts of the ALS (Advanced Light Source) [1] storage ring and booster synchrotron lattices. The brightness and lifetime requirements for the photon beams to be produced by the storage ring imply very tight tolerances for the fields of these magnets [2], [1]. The main concern here lies on the magnet-to-magnet reproducibility of the fundamental excitation; the higher-order multipole errors are largely determined by the given pole shapes optimized in earlier investigations of engineering models of each basic magnet type [3].

All production series magnets were measured under d.c. conditions even though the booster magnets will be ramped with $1 \mathrm{~Hz}$ repetition rate. The dynamical behavior of the booster bend magnet engineering model, however, had previously been studied at ramping frequencies of 1,2 , and 10 $\mathrm{Hz}$ [4]. The multipole magnets for both rings were measured using rotating, integrating coils. For the storage ring gradient magnets an elaborate Hall probe scan had to be performed to ascertain sufficient accuracy for the measurements of their quadrupole components. In addition, a rolating coil was used for this magnet type to evaluate the higher-order multipole fields. The booster bend magnets were magnetically measured using mobile integral coils to obtain relative uniformity data and determine the relative change in effective length as a function of the excitation value; the absolute effective lengths were deduced from Hall probe scans of the engineering model and mechanical length and gap width data of each magnet [5].

The evaluation procedures and results will be discussed for each magnet type. Note that all booster magnets have closed yokes whereas all storage ring magnets have C-type yokes to provide apertures for the synchrotron radiation. All nominal magnet parameters given below refer to the nominal beam energy of $1.5 \mathrm{GeV}$.

\section{BOOSTER DIPOLES}

The booster bend magnet is a curved, flat-field dipole; its main nominal parameers are: bend angle, $15^{\circ}$; magnetic length, $1.050 \mathrm{~m}$; pole tip field, $1.248 \mathrm{~T}$ at $744 \mathrm{~A}$ excitation current; rms field integral tolerance, $1 \times 10^{-3}$. The most critical quality U.S. Government work not protected by U.S. Copyright. of this magnet type is its integrated field. The measurement system used to evaluate the absolute field length of the engineering model [4], however, did not provide enough accuracy to compare the individual production magnets in this respect. Instead, the mechanical data of magnet core length and gap width [5] were chosen to determine the relative bending power of every production magnet from the length-over-gap ratio. This procedure is justified by the elaborate lamination sorting process established to assure good magnet-to-magnet field reproducibility. The statistics for the 25 manufactured magnets (including one spare) are listed in Table 1.

Table 1

Booster Dipoles Mechanical Qualities, Relative Deviations

\begin{tabular}{|l|c|c|c|}
\hline & Length & Gap & Length/Gap \\
\hline$\sigma_{\text {rel }}$ & 0.00021 & 0.00033 & 0.00042 \\
\hline Worst & -0.00042 & 0.00068 & -0.00080 \\
\hline
\end{tabular}

The data in Table 1 show that the derived variations in the integrated fields of the booster bend magnets should be well within the specified tolerance of $11 \times 10^{-3} \mathrm{rms}$. Therefore, the magnets could be placed in the booster lattice without observing any particular positioning order.

To characterize the field uniformity of all bend magnets a statistical overview of their sextupole coefficients, $\mathrm{k} 3$, is given in Table 2. The definition for $\mathrm{k} 3$ is:

$$
\mathrm{k}_{3}=\frac{1}{\mathrm{~B}_{1} \rho} \frac{\mathrm{d}^{2} \mathrm{~B}_{3}}{\mathrm{dx}^{2}}
$$

with $B_{1}$, dipole field, $1.248 \mathrm{~T} ; \rho$, beam curvature radius, $4.0107 \mathrm{~m}$; and $B_{3}$, Sextupole field, as measured by a bucked pair of integrating coils.

Table 2

Booster Dipoles Sextupole Coefficients

\begin{tabular}{|l|c|}
\hline Average $\mathrm{k}_{3}\left[1 / \mathrm{m}^{3}\right]$ & 0.27 \\
\hline$\sigma\left(\mathrm{k}_{3}\right)\left[1 / \mathrm{m}^{3}\right]$ & 0.041 \\
\hline Worst Dev. $\left[1 / \mathrm{m}^{3}\right]$ & 0.156 \\
\hline
\end{tabular}

All actual sextupole magnets together provide an integral field that is 55 times stronger than the sum of the random components of the integrated sextupole fields created by all dipoles (see Table 4); this ratio is acceptable for the booster synchrotron.

\section{BOOSTER QUADRUPOLES}

There are two types of booster quadrupoles: focusing (QF) and defocusing (QD) with 0.282 and $0.182 \mathrm{~m}$ effective lengths, respectively, and identical pole contours. The nominal gradi- 
ents are 15.91 and $16.37 \mathrm{~T} / \mathrm{m}$, with maximum fields at the pole tips reaching 0.517 and $0.532 \mathrm{~T}$. The tolerance for magnet-to-magnet gradient variations was set to $2 \times 10^{-3} \mathrm{~ms}$.

The magnets were measured using a rotating, integrating coil in a bucked mode to evaluate higher-order multipoles. Integrated gradients were evaluated by comparing each of the magnets to one standard magnet for each type, using the rotating coil in an unbucked mode. The absolute reference was established by combining the electrical parameters of the measurement system and of the coil geometry with averaged data gathered for each of the two standard magnets. The resulting uncertainty of about $1 \%$ in the absolute gradients does not cause any problem for booster operation as long as the magnet-to-magnet reproducibility is within tolerance.

Table 3 gives the statistics for the fundamental and first allowed higher multipole strengths. The fundamental is listed in the form of the transfer function of the integrated gradient, $\mathrm{k}_{2} \mathrm{~L} / \mathrm{I}$, with I, excitation current; L, effective length; and

$$
\mathrm{k}_{2}=\frac{1}{\mathrm{~B}_{1} \rho} \frac{\mathrm{dB}_{2}}{\mathrm{dx}}
$$

The nominal currents are $418 \mathrm{~A}$ for the focusing and $430 \mathrm{~A}$ for the defocusing quadrupole. The dodecapole strength is expressed as the ratio of the integrated dodecapole field over the integrated quadrupole field, both at $30 \mathrm{~mm}$ radius. The entries for standard deviations and worst cases are given as relative quantities, $\sigma_{\mathrm{rel}}$, in units of the average values. The measured average values and individual data allowed us to qualify all measured quadrupoles for installation in the booster ring.

Table 3

Booster Quadrupoles

\begin{tabular}{|l|c|c|c|}
\hline & Average & $\sigma_{\text {rel }}$ & worst \\
\hline QF Fund. $\mathrm{k}_{2} \mathrm{l}, / \mathrm{I}\left[(\mathrm{Am})^{-1}\right]$ & 0.002261 & 0.0011 & -0.0018 \\
\hline QF Dodecapole $\mathrm{B}_{6} / \mathrm{B}_{2}$ & 0.000286 & 0.019 & 0.039 \\
\hline QD Fund. $\mathrm{k}_{2} \mathrm{~L} / \mathrm{I}\left[(\mathrm{Am})^{-1}\right]$ & 0.001465 & 0.0014 & 0.0020 \\
\hline QD Dodecapole $\mathrm{B}_{6} / \mathrm{B}_{2}$ & 0.000431 & 0.025 & 0.048 \\
\hline
\end{tabular}

\section{BOOSTER SEXTUPOLES}

Focusing and defocusing booster sextupoles are identical in design with the following main nominal parameters: effective length, $0.121 \mathrm{~m}$; sextupole coefficient, up to $55.5 \mathrm{~T} / \mathrm{m}^{2}$; pole tip field, up to $0.068 \mathrm{~T}$ at $4.8 \mathrm{~A}$. Table 4 gives an overview of the fundamental and first allowed higher multipole strengths; the explanations given in the previous section on quadrupoles apply with due modifications. The strength parameter $\mathrm{k}_{3}$ is defined in Eq. 1. Again, the data proved good enough for all sextupoles to be installed in the booster ring.

Table 4

Booster Sextupoles

\begin{tabular}{|l|c|c|c|}
\hline & Average & $\sigma_{\text {rel }}$ & Worst \\
\hline $\mathrm{k}_{3} \mathrm{~L} / 1\left[\mathrm{~A}^{-1} \mathrm{~m}^{-2}\right]$ & 0.575 & 0.0010 & -0.0018 \\
\hline Octocaidecapole & 0.00447 & 0.019 & 0.064 \\
\hline
\end{tabular}

\section{STORAGE RING GRADIENT MAGNETS}

The storage ring bend magnet is a straight, parallel-ended combined-function magnet with the following main parameters: bend angle, $10^{\circ}$; magnetic length, $0.865 \mathrm{~m}$; pole tip field, $1.047 \mathrm{~T}$ and field gradient, $-4.036 \mathrm{~T} / \mathrm{m}$, both at $700 \mathrm{~A}$; rms field integral tolerance, $1 \times 10^{-3}$. As main characteristic data, the integrated dipole field, the integrated gradient, the core field, and the core gradient were evaluated at 480,700 , and 910 A excitation current. The central part of the magnet, extending to $\pm 250 \mathrm{~mm}$ from the mid plane, is here defined as the magnet core. The Hall probe scan was taken over an area of $1800 \times 60$ $\mathrm{mm}^{2}$, with $10 \mathrm{~mm}$ distance between measurement points in both directions. The raw data were adjusted for the actual excitation currents and the offset of every measurement point against its ideal transverse location. The Hall probe holder was aligned to the magnet with $25 \mu \mathrm{m}$ precision using a laser system. Field integration was performed numerically, with trapezoidal approximation. Statistics at the nominal $1.5 \mathrm{GeV}$ condition (700 A excitation current) for the eight magnets so far evaluated are given in Table 5.

The higher-order multipoles were obtained from three magnets, each measured twice using an integrating, rotating coil; the listed values represent the ratios of the integrated fields at $20 \mathrm{~mm}$ transverse position over the integrated dipole field components. Statistics for the three strongest higher multipoles are given in Table 6 . Due to the gradient design, the magnets have effective edge angles of $2^{\circ}$ (larger at the side with the wider gap) that somewhat reduce the defocusing action of the gradient and also a notable curvaturc (sec Fig. 1).

Table 5

Storage Ring Bend Magnets Fundamental Parameters

\begin{tabular}{|l|c|c|c|c|}
\hline & $\begin{array}{c}\mathrm{B} \mathrm{L} \\
{[\mathrm{Tm}]}\end{array}$ & $\begin{array}{c}\mathrm{dB} / \mathrm{dx} \mathrm{L} \\
{[\mathrm{T}]}\end{array}$ & $\begin{array}{c}\mathrm{B} \\
{[\mathrm{T}]}\end{array}$ & $\begin{array}{c}\mathrm{dB} / \mathrm{dx} \\
{[\mathrm{T} / \mathrm{m}]}\end{array}$ \\
\hline Average & 0.9057 & -3.419 & 1.0469 & -4.038 \\
\hline$\sigma_{\text {rel }}\left[10^{-3}\right]$ & 0.42 & 0.66 & 0.25 & 0.67 \\
\hline Relat. Worst $\left[10^{-3}\right]$ & 0.88 & 1.02 & 0.39 & 1.02 \\
\hline
\end{tabular}

Table 6

Storage Ring Bend Magnets Higher Multipoles

\begin{tabular}{|l|c|c|c|}
\hline & Average & $\sigma_{\text {rel }}$ & Rel. Worst \\
\hline Sextupole $\mathrm{B}_{3} / \mathrm{B}_{1}$ & 0.00022 & 0.07 & 0.10 \\
\hline Octupole $\mathrm{B}_{4} / \mathrm{B}_{1}$ & 0.00003 & 1.0 & 1.3 \\
\hline Decapole $\mathrm{B}_{5} / \mathrm{B}_{1}$ & 0.00006 & 0.2 & 0.4 \\
\hline
\end{tabular}

\section{STORAGE RING QUADRUPOLES}

There are three families of quadrupoles with identical crosssectional design but different effective lengths: QFA (focusing), $0.445 \mathrm{~m}$; QF (focusing), $0.350 \mathrm{~m}$; and QD (defocusing), $0.200 \mathrm{~m}$. The nominal gradients are $15 \mathrm{~T} / \mathrm{m}$ at $400 \mathrm{~A}$ excitation current with $0.621 \mathrm{~T}$ pole tip field for QFA and 13.4 T/m at $89 \mathrm{~A}$ with $0.434 \mathrm{~T}$ pole tip field for $\mathrm{QF}$ and $\mathrm{QD}$. The magnet-to-magnet tolerance for the integrated fields is $1 \times 10^{-3}$ in all three cases. For the time being, only QFA-type quadrupoles have been measured in a sufficient number to allow a quality assessment in terms of this tolerance. 


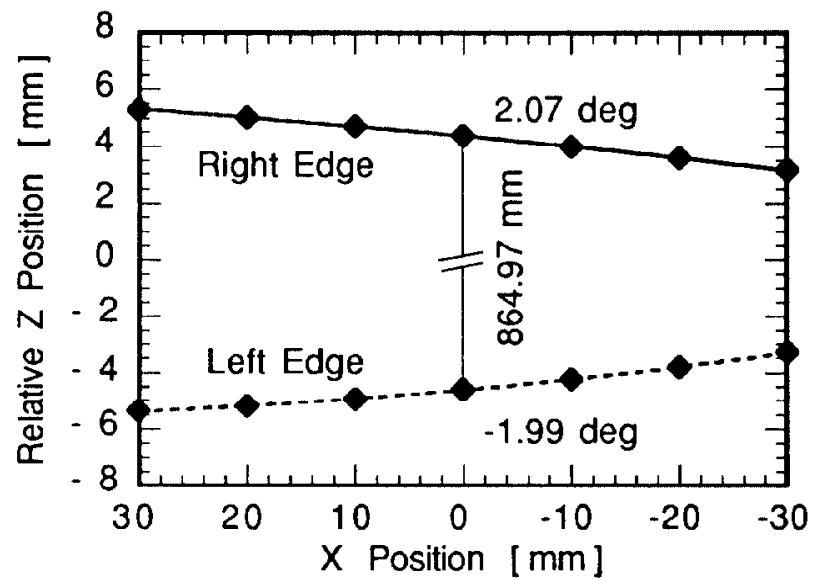

Figure 1. Effective edges of storage ring bend magnet $\# 16$. The positive $X$ direction points radially outwards; the beam travels in positive $Z$ direction. The data are fitted by parabolas.

The first data that were taken, however, showed disappointingly bad magnet-to-magnet reproducibility, in spite of the elaborate lamination sorting process and tight manufacturing tolerances imposed on all storage ring quadrupoles. For 12 measured magnets, the standard deviation of the integrated fundamentals amounted to $1.8 \times 10^{-3}$ with a worst case of $3.3 \times 10^{-3}$. An investigation pointed to short-time current fluctuations in the power supply as cause; improvements of the supply stability and the measurement procedure, with more frequent readings of the current, are underway.

\section{STORAGE RING SEXTUPOLES}

The storage ring sextupole is a multifunction magnet with identical design for focusing and defocusing [1]. The nominal fundamental parameters for $1.5 \mathrm{GeV}$ beam energy are: pole tip field $0.484 \mathrm{~T}\left(395 \mathrm{~T} / \mathrm{m}^{2}\right)$ at 290 A excitation with $0.2 \mathrm{~m}$ effective length. Apart from the sextupole field, this magnet

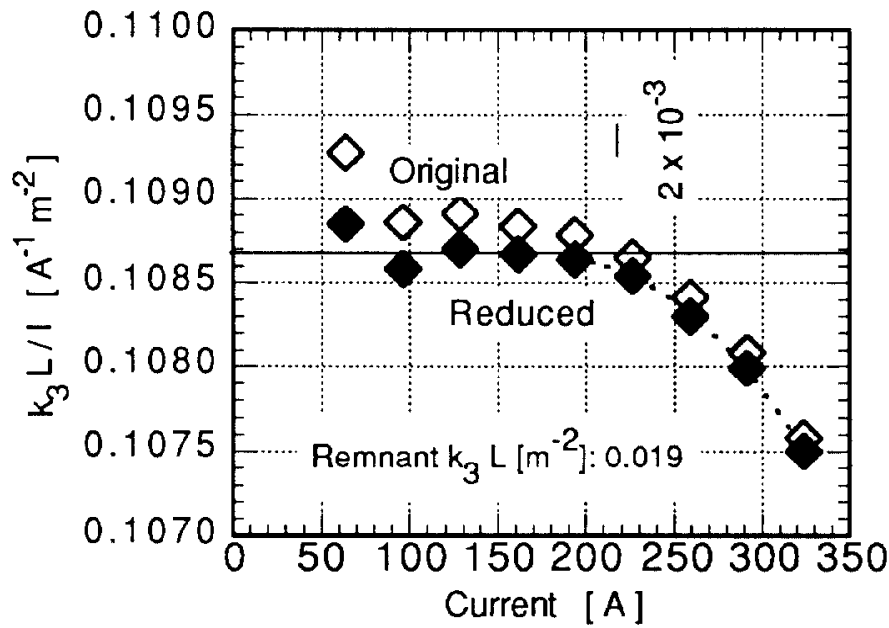

Figure 2. Reduced transfer function of storage ring sextupole \#34. The straight line marks the average value of the first five points, and a parabola (dashed curve) is fitted to the saturation part. can produce horizontal and vertical steering fields and a skew quadrupole field by exciting different poles with separate windings, but these "trim" functions were not quantitatively analyzed for the production series. The excitation curves are given in the form of reduced transfer functions after subtracting the remnant fields (see Fig. 2). Statistics are listed in Table 7; the fundamental strength parameter $k_{3}$ is defined in Eq. (1). The slight excess over the set standard deviation tolerance of $1 \times 10^{-3}$ for the fundamental was judged acceptable, and the sixteen magnets so far measured were qualified for installation.

Table 7.

Storage Ring Sextupoles

\begin{tabular}{|l|c|c|c|}
\hline & Average & $\sigma_{\text {rel }}$ & Worst \\
\hline Fund. $\mathbf{k}_{\mathbf{3}} \mathrm{L} / \mathrm{I}\left[\mathrm{A}^{-1} \mathrm{~m}^{-2}\right]$ & 0.10869 & 0.0012 & 0.0022 \\
\hline Remnant $\left.\mathrm{k}_{\mathbf{3}} \mathrm{L}^{-2}\right]$ & 0.0242 & 0.20 & -0.44 \\
\hline
\end{tabular}

\section{ACKNOWLEDGMENTS}

The measurements on which the presented analysis is based where performed at LBL by T. Harten, D. Van Dyke, and F. Zucca, under the guidance of J. Tanabe, further by D. Nelson, S. Marks, and K. Luchini. The data acquisition codes used during the measurements were created by S. Marks and K. Luchini, with a contribution by $J$. Bengtsson. R. Alvis assisted in the evaluation of the raw data. Thanks are also due to A. Jackson for many discussions and advice in defining the evaluation procedures.

This work was supported by the Director, Office of Energy Research, Office of Basic Energy Sciences, Material Sciences Division, U.S. Department of Energy, under Contract No. DEAC03-76SF00098.

\section{REFERENCES}

1. "1-2 GeV Synchrotron Radiation Source, Conceptual Design Report," LBL Pub. 5172 Rev., LBL Berkeley, 1986.

2. A. Jackson, "Magnetic Field Tolerances in the ALS Storage Ring," ALS LSAP-56, LBL Berkeley, 1989.

3. J. Tanabe et al., "Fabrication and Test of Prototype Ring Magnets for the ALS," IEEE 89CH2669-0, vol.1, pp. 566-568, 1989.

4. R. Keller et al., "Magnetic Properties of the ALS Booster Synchrotron Engineering Model Magnets," IEEE 89CH 2669-0, vol.3, pp. 1966-1968, 1989.

5. R. Joens and E. Hoyer, "Advanced Light Source - Booster Dipole Magnet, Summary of Magnet Mechanical Measurements," ALS LSME-234, LBL Berkeley, 1989. 